J. Lake Sci. (湖泊科学) , 2011, 23(4): 513-519

http: //www. jlakes. org. E-mail : jlakes@niglas.ac.cn

(c) 2011 by Journal of Lake Sciences

\title{
太湖微囊藻毒素时空分布特征及与环境因子的关系
}

\author{
王经结,杨 佳, 鲜啟鸣 ${ }^{* *}$, 钱 新, 李正鬼, 耿金菊, 孙 成 \\ (南京大学环境学院污染控制与资源化研究国家重点实验室,南京 210093)
}

\begin{abstract}
摘 要: 采用高效液相色谱法 (HPLC) 对太湖水体中溶解态微囊藻毒素 (MC-LR; MC-RR) 浓度进行检测, 探讨微囊藻毒 素浓度的时空变化及其与氮、磷、总有机碳、蓝藻生物量以及温度等湖泊环境因子之间的关系. 结果表明: 微囊藻毒素表 底层浓度基本一致; 在一天之内的变化没有明显规律; 冬季微囊藻毒素浓度较高; 位于竺山湾和西部沿岸地区的两个点 (N5 和 N1) 的微囊藻毒素浓度在一年之中变动较大; MC-LR 浓度一般大于 MC-RR, 但在 8,9 两月出现相反的结果. 水中 溶解性微囊藻毒素与各环境因子的相关性分析表明微囊藻毒素与总氮呈极显著正相关, 与总磷无显著相关性. 在蓝藻暴 发期, MC-LR 与总氮、总磷、SS、总有机碳、蓝藻生物量呈极显著正相关; MC-RR 与所测的环境因子皆无显著相关性.
\end{abstract}

关键词: 微囊藻毒素;太湖; 环境因子;相关性

\section{Characteristic of microcystin distributions and its relationships with environmental factors in Lake Taihu}

WANG Jingjie, YANG Jia, XIAN Qiming, QIAN Xin, LI Zhengkui, GENG Jinju \& SUN Cheng (State Key Laboratory of Pollution Control and Resource Reuse, School of the Environment, Nanjing University, Nanjing 210093, P. R. China)

\begin{abstract}
In this paper, the high performance liquid chromatography (HPLC) was used to monitor the spatial and temporal distribution of dissolved Microcystin-LR and Microcystin-RR in Lake Taihu, and its relationship with physiochemical factors of the lake were also discussed. The results showed that the concentrations of microcystin(MCs) were not different between surface and bottom water in a day, and the highest concentration of microcystins occurred in winter. Concentrations of the MCs changed much during one year in the sampling sites N5 and N1 which located in Zhushan Bay and the western part of Lake Taihu, respectively. Generally, the concentration of the MC-LR was higher than MC-RR for the year in average while the opposite results appeared in individual month of August and September. Correlation analysis showed that MC-LR was significantly positive correlated with TN, but did not show correlation with TP. During the period of water bloom of cyanobacteria, levels of the MC-LR were significantly positive correlated with $\mathrm{TP}, \mathrm{TN}, \mathrm{UV}_{254}$, SS, total organic carbon and biomass of cyanobacteria, whereas MC-RR was not significant correlated with all the environmental factors in the statistical test.
\end{abstract}

Keywords: Microcystin; Lake Taihu; environmental factors; correlation

近年来, 随着经济的迅猛发展, 水体富营养化程度加剧, 有害蓝藻水华发生日趋频繁, 这引起国内外 研究人员的普遍关注. 蓝藻水华暴发不仅造成水质下降, 而且微囊藻 (Microcystis)、鱼腥藻 (Anabaena)、颤 藻 (Oscillatoria) 等还向水体释放微囊藻毒素 (Microcystins, MCs), 危害水生生态系统的安全 ${ }^{[1]}$. MCs 是一 类单环七肽化合物, 具有多种同分异构体 ${ }^{[2]}$, 主要类型有 3 种, 分别是 MC-LR、MC-RR 和 MC-YR $(L 、 R 、 Y$ 分别代表亮氨酸、精氨酸和酪氨酸), 其中 MC-LR 和 MC-RR 所占比例较高, 是主要研究对象 ${ }^{[34]}$. MCs 具 有肝脏毒性和肿瘤促进作用 ${ }^{[5]}$, 它不仅对水生生物产生毒害作用,还可能通过饮用水和食物链的生物 富集危害人类健康. 影响蓝藻生长及产毒能力的环境因素众多, 不同时间不同藻的产毒能力不同, 产

* 国家“973”计划项目 (2008CB418003) 和国家水体污染控制与治理科技重大专项 (008ZX07421 - 002) 项目联合资助. 2010-08-16 收稿;2010-12-21 收修改稿. 王经结,女,1985 年生, 硕士研究生;E-mail : wangjingjie66@ gmail. com.

** 通讯作者;E-mail:xianqm@ nju. edu.cn. 
生的毒素种类也不一定相同 ${ }^{[6]}$, 因此要了解 MCs 在自然水体中的分布需要综合考虑各种环境因子的 作用.

太湖是我国第三大淡水湖, 现有水面面积 $2338 \mathrm{~km}^{2}$, 是典型的浅水湖泊, 同时也是蓝藻水华多发的湖 泊. 本研究选取太湖作为研究对象, 对水中 MC-LR 和 MC-RR 进行为期一年的监测, 研究藻毒素在水体中 的时空分布特征及影响因素, 以探索 MCs 在自然水体中的形成规律, 为研究藻毒素的生态风险提供理论 依据.

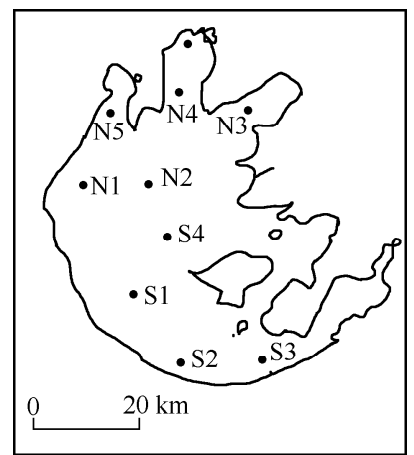

图 1 采样点位置

Fig. 1 Sampling sites in Lake Taihu

\section{1 材料与方法}

\section{1 采样点布设}

根据太湖湖底地形、湖面面积、湖区地理位置、富营养状况及主要分 布特征等, 在太湖选取了九个点 (图 1 ), 北湖五个点, 南湖四个点, 这九个 点覆盖整个太湖, 并具有较好的代表性.

\section{2 样品采集}

2009 年 2 月到 2009 年 5 月, 2009 年 8 月到 2009 年 9 每月采样一次, 2009 年 11 月和 2010 年 1 月各采样一次. 在七月初蓝藻水华高峰期选择 三天对 $\mathrm{N} 1, \mathrm{~N} 2$ 两点进行重点监测, 每天采样五次, 采样时间分别为 8:00, $10: 00,12: 00,14: 00,16: 00$. 使用有机玻璃采水器, 采集水面下 $0.5 \mathrm{~m}$ (表 层) 和 $2 \mathrm{~m}$ (底层) 处的水样, 除 $\mathrm{N} 1, \mathrm{~N} 2$ 分层储存外, 其他点均将水样混匀 后再储存. 采样船只采用湖中地物结合 GPS 定位仪进行定位. 待所有的样 品采集完毕后, 立即送回实验室, 根据不同监测指标的具体要求保存样 品, 待测.

\section{3 水质指标的测定方法}

1.3 .1 常规指标的分析测定 悬浮质 $(\mathrm{SS})$ 为 $103^{\circ} \mathrm{C}-105^{\circ} \mathrm{C}$ 烘干的不可滤残渣 (悬浮物 $)^{[7]}$, 水温采用 Aaq1183 水质自动测定仪测定, 总磷 (TP)、总溶解态磷 (TSP)、溶解态反应磷 ( SRP) 采用过硫酸钾一钼锑抗分 光光度法, 总氮 $(\mathrm{TN})$ 采用过硫酸钾氧化一紫外分光光度法, 亚硝态氮 $\left(\mathrm{NO}_{2}-\mathrm{N}\right)$ 采用 $\mathrm{N}-(1-$ 菜基 $)-乙 二$ 二胺光 度法, 氨氮 $\left(\mathrm{NH}_{3}-\mathrm{N}\right)$ 采用纳氏试剂光度法 ${ }^{[7]}$, 硝态氮 $\left(\mathrm{NO}_{3}-\mathrm{N}\right)$ 采用球香草酚分光光度法, 总有机碳 $(\mathrm{TOC})$ 采 用过 $0.45 \mu \mathrm{m}$ 滤膜后 Aurora1030D 湿法 ( TC-IC 模式) 测定, UV254 采用过 $0.45 \mu \mathrm{m}$ 滤膜在 $254 \mathrm{~nm}$ 下分光光度 法测定 ${ }^{[8]}$; 蓝藻生物量经鲁哥试剂固定、沉淀、浓缩后高倍显微镜计数得到 ${ }^{[9]}$.

1.3 .2 微囊藻毒素的分析测定 量取 $1 \mathrm{~L}$ 经 $0.45 \mu \mathrm{m}$ 滤膜过滤 (水体中浮游生物和悬浮物含量较大时可先用 500 目不锈钢篮过滤) 的水样置于棕色瓶中. 连接 SPE 固相萃取装置将水样通过预先活化过的 Sep-pak 小柱 ( 控制流速为 $8-10 \mathrm{ml} / \mathrm{min}$ ). 富集完毕后, 依次用 $10 \mathrm{ml}$ 纯水和 $10 \mathrm{ml}$ 体积分数为 $20 \%$ 的甲醇溶液淋洗固相萃 取小柱. 再用 $10 \mathrm{ml}$ 含 $1 \% 0$ 三氟乙酸的甲醇溶液洗脱, 洗脱液经氮吹后用 $50 \%$ 的甲醇定容到 $500 \mu l$, 待液相测 定. HPLC 测定条件: 进样量 $20 \mu \mathrm{l}$; 流动相为 $57: 43$ 的甲醇与磷酸盐缓冲液; 柱温为 $40^{\circ} \mathrm{C}$; 流速为 $1 \mathrm{ml} / \mathrm{min}$; 检 测波长为 $238 \mathrm{~nm}$. MC-LR 和 MC-RR 标准样品均购自中科院武汉水生生物研究所(纯度达 $99 \%$ ).

\section{4 统计方法}

将所有数据在 Excel 中建立数据库, 用 SPSS 15.0 对水质数据进行 Spearman 相关性分析以及逐步回归 分析, 借助 SigmaPlot 10.0 软件进行图表处理.

\section{2 结果与讨论}

\section{1 太湖水体中 MCs 的分布}

2.1.1 MCs 浓度的深度差异 MCs 在表底层的分布具有很好的一致性(图 2). 这一规律在 7 月份蓝藻暴发 期的连续监测中也得到体现. 这是由于太湖平均深度较浅 ( $<2 \mathrm{~m})$, 难以形成分层现象, 且面积较大 $\left(2338 \mathrm{~km}^{2}\right)$, 受风浪扰动较大, 易混合均匀; 同时 MCs 为极性分子, 在水中具有较大的溶解度 $(>1 \mathrm{~g} / \mathrm{L})$, 比较 容易扩散. 

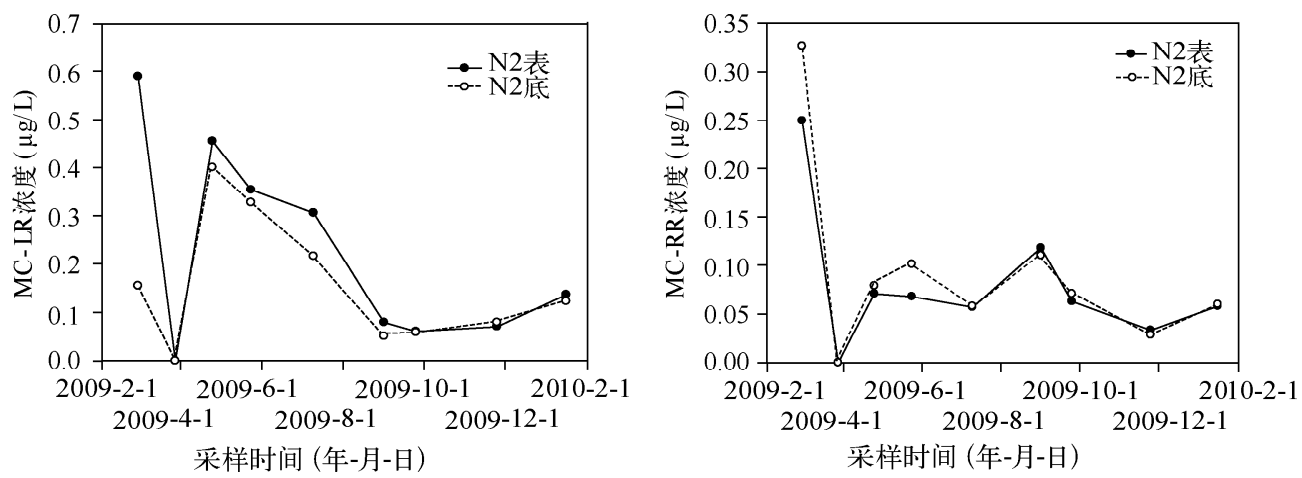

图 $2 \mathrm{MCs}$ 在水体表底层的浓度变化

Fig. 2 The distribution of MCs in the bottom and surface of water

2.1.2 MCs 浓度的空间分布 对于 MC-LR, 其空间分布特征表现为: S4、S1 > S2 > S3, N5 > N2、N4>N3(图 3 ). 而 MC-RR 的空间分布特征为: $\mathrm{S} 2>\mathrm{S} 4>\mathrm{S} 1 、 \mathrm{~S} 3, \mathrm{~N} 1>\mathrm{N} 2 、 \mathrm{~N} 4>\mathrm{N} 3$ (图 3). 无论 MC-LR 还是 MC-RR, 浓度 均低于 $\mathrm{WHO}$ 和我国生活饮用水推荐的标准 $(1 \mu \mathrm{g} / \mathrm{L})$. MCs 在南北湖区之间未显示出区域性分布差异,在 N5 点显示出年际波动, MC-RR 表现的尤其明显. 09 年 4 月份低于检测限, 而 2009 年 9 月份浓度高达 $0.7 \mu \mathrm{g} / \mathrm{L}$ (图 4). 在蓝藻暴发之前 N1 点的 MC-LR 浓度低于 N2 点, 暴发后浓度高于 N2 点, 说明蓝藻暴发促进了 MCLR 在 N1 点的积累. 这可能是因为 N1 点靠近西北岸边,夏季太湖地区以东南风为主,使得藻在 N1 点聚集, 从而导致该点浓度的增加. 同时 $\mathrm{N} 1$ 点位于东氿湖下方,上游来水的影响较大. 所以 $\mathrm{N} 1$ 浓度的高低受释放到 水中的藻毒素含量以及水量的双重影响. 九个点中只有 N5 和 N1 处于 2006 年水质调查 ${ }^{[10]}$ 的劣 V 类区域之 中, 其中是否存在关系, 还需要进一步调查研究.
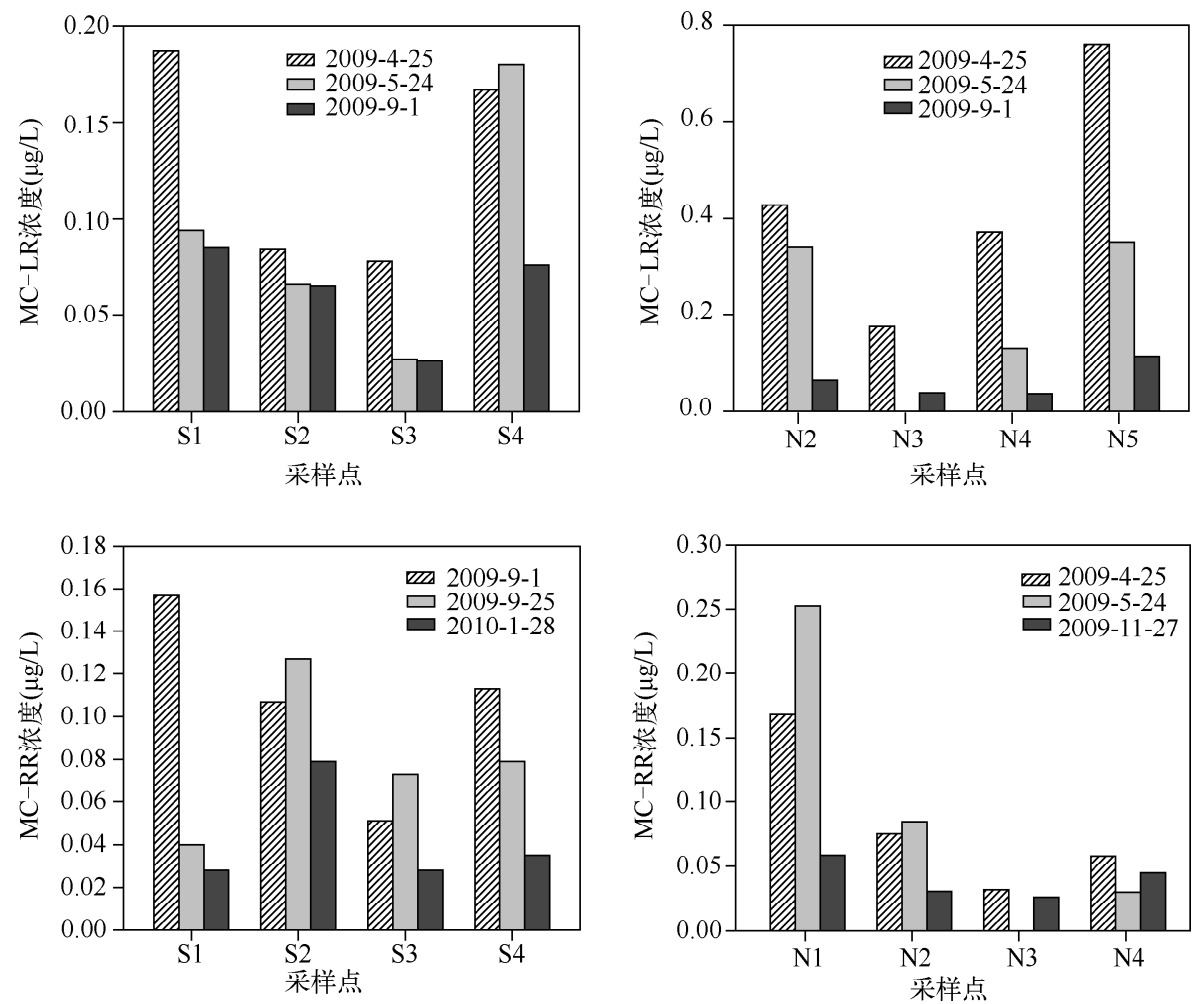

图 3 MCs 在太湖的分布状况

Fig. 3 The distribution of MCs in Lake Taihu 


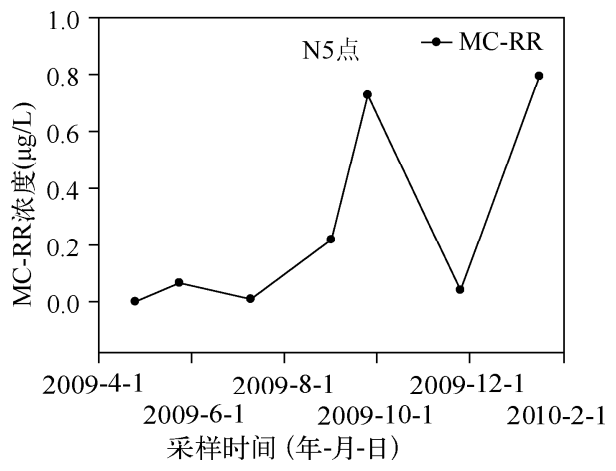

图 4 N5 点 MC-RR 的浓度

Fig. 4 Concentration of MC-RR in site N5

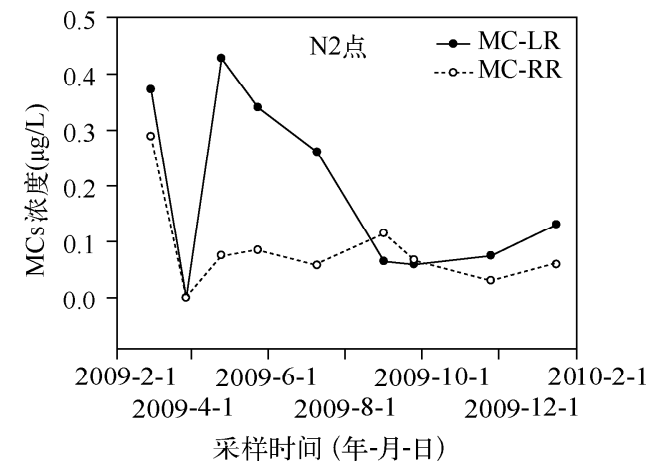

图 $5 \mathrm{MCs}$ 随时间的变化

Fig. 5 The changes of MCs with time

2.1.3 MCs 的周年变化 冬季 MCs 浓度较大, 各点的 MCs 浓度从 2009 年 2 月至 4 月呈增加趋势; 从 2009 年 4 月至 7 月浓度较高但呈减小的趋势, 从 2009 年 11 月至 2010 年 4 月呈增加趋势 (图 5 ). 蓝藻的数量从 4 、5 月份开始增加, 同时伴随着藻的死亡, 向水体中分泌藻毒素, 但是自然水体中微囊藻毒素的去除速度也很 快 $^{[11]}$, 且降水增多, 在一定程度上对藻毒素具有稀释的作用. 所以在 7 月份蓝藻暴发式生长之前毒素浓度仍 然呈增加趋势. 水量对水体中 MCs 的影响占主要地位. 10 月份之后藻的密度开始下降, 同时太湖流域降水 减少, 水量的减少对毒素起到一个浓缩的效果. 另外, 冬季微生物以及水生生物的活性降低, 对藻毒素的降 解作用减小. 有研究表明在自然环境中底泥沉积物中细菌对微囊藻毒素的生物降解起到关键的作用 ${ }^{[12]}$; 浅 水湖泊浊度高, 光降解的贡献很小, 因此毒素以积累为主, 所以到四月为止毒素仍然呈增加的趋势. 从 2009 年 7 月至 11 月, MC-LR 浓度缓慢增加, 但是 MC-RR 浓度有一个明显先增加后减小的过程, 并且在 $8 、 9$ 月份 出现 MC-RR 浓度大于 MC-LR 的现象. MCs 是一类受产毒基因调控, 在胞内代谢产生的毒素. 有研究表明在 与其它藻类共生的情况下产毒微囊藻更有竞争优势 ${ }^{[13]}$, 在蓝藻暴发期微囊藻产毒基因的丰度显著增 加 ${ }^{[14-15]}$, 因此处于暴发期的蓝藻可能合成更多的毒素. 但微囊藻毒素主要集中在细胞的类囊体核区, 很少存 在于细胞壁和峭壁上 ${ }^{[16]}$, 只有当藻细胞死亡、破裂时才会释放到水体中 ${ }^{[17]}$. 但是即使藻已进人稳定期或达 到很大的密度也可能只有很少部分的藻细胞死亡 ${ }^{[18-20]}$. 自然水体中, 微囊藻毒素一般在蓝藻大量衰亡的季 节才会大量释放到水柱中 ${ }^{[11]}$, 因此 MCs 浓度的增加在蓝藻暴发之后. 微囊藻水华发展过程中, 存在多种基 因型共存和微囊藻不同基因型的演替 ${ }^{[21]}$, 可能在蓝藻暴发前期合成 MC-RR 的基因种类较多后期较少, 或是 受其它环境因子 (如光照、温度、氮、磷等) 的影响, 出现 MC-RR 浓度先增加后减小的现象. 迄今为止, 关于产 生不同藻毒素的原因 (是由基因种类决定还是受环境因子调控,或是两者共同作用) 鲜有报道. 要解释这种 现象, 还需要进一步研究.

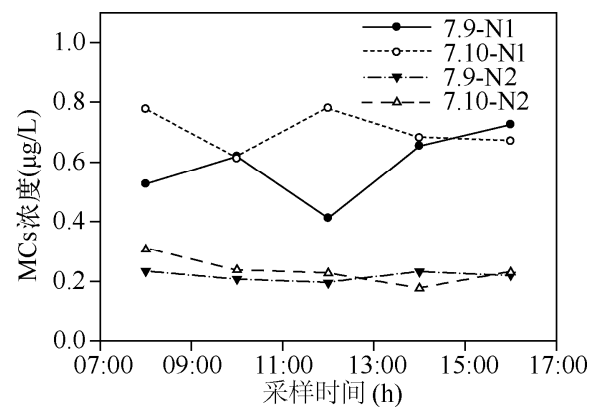

图 $6 \mathrm{MCs}$ 在一天之内的变化

Fig. 6 The changes of MCs within one day
2.1.4 MCs -天之内的变化 对 7 月份集中采样的数据分 析发现 MCs 的浓度比较稳定, 波动较小, 一天之内的变化规 律不明显(图 6). MCs 主要咜存在细胞内, 细胞死亡后毒素 才会释放到水体中. 实验室研究发现, 处于对数生长期的微 囊藻培养物中至多能释放 10\%-20\% 的毒素到培养基中; 进 人稳定期时, 随着藻死亡数量的增加, 培养基中毒素的比例 会有所增加. 但是在蓝藻的生长周期里, 藻的死亡数量只占 很少部分 ${ }^{[18-20]}$. 因此, 在蓝藻生长周期里, 释放到水体中的毒 素含量是有限的. 在自然环境中, 微囊藻毒素的大量释放一 般发生在水华衰亡的时期. 因此在水华暴发期, 一天之内释 放到水柱中的藻毒素的量很小且相对较稳定. 并且, 在蓝藻 暴发期, 藻密度较大, 水体透明度较低, 光降解的作用非常有 
限. 底泥中细菌可能在自然环境藻毒素的降解过程中起着关键的作用 ${ }^{[12]}$, 源于底泥中的细菌对水柱中毒素 的降解与水柱中藻毒素的释放维持着动态平衡.

\subsection{MCs 与环境因子的相关性分析}

氮是藻类生长的必需营养元素, 同时氮作为一种必需的元素参与藻毒素的合成 ${ }^{[22]}$, 因而氮缺乏 $(<12.53 \mathrm{mg} / \mathrm{L})$ 不仅限制藻的生长还抑制藻毒素的分泌 ${ }^{[23]}$. 本研究水体中 TN 的均值为 $3.04 \mathrm{mg} / \mathrm{L}$, 浓度较 低, 可能抑制藻毒素的分泌. 全年相关性分析结果 (表 1) 也表明水中溶解性 MC-LR 与 TN 呈极显著正相关, 但是 MC-RR 与 TN 无显著相关性. 不同月份 MCs 与 TN 相关性分析表明, 大部分时间 MC-LR、MC-RR 均与 $\mathrm{TN}$ 呈极显著正相关; MC-LR 与 TN 相关性偶尔有所变动; MC-RR 与 TN 在 7 月份蓝藻暴发到 9 月份没有显 著相关性 (表 2), 这与 MC-RR 在此期间浓度增加有关系. 总体来说, MCs 与 TN 具有很好的相关性, 这与之 前的报道一致 ${ }^{[24-25]}$. 全年相关性分析结果 (表 1) 表明 TP 与水中溶解性 MC-LR 呈极显著正相关, 与 MC-RR 无显著相关性. 而 TP 与 MC-LR 和 MC-RR 在所有月份均无显著相关性. 因而 TP 与 MC-LR 在全年表现出的 极显著正相关性可能由二者的季节变动存在一致性导致的,二者没有直接的关系. 关于 TP 对 MCs 的影响至 今也没有一致的研究结论. Sivonen ${ }^{[19]}$ 认为当磷的浓度在 $0.1-0.4 \mathrm{mg} / \mathrm{L}$ 范围内 MCs 含量与磷浓度呈正相 关, 但更高浓度的磷与 MCs 浓度相关性不明显. Utkilen 等 ${ }^{[26]}$ 认为磷对微囊藻毒素的产生没有影响. 也有研 究表明微囊藻的产毒能力随着磷浓度的减小而增加 ${ }^{[27-28]}$. 由于 TN/TP 波动较大, 在 $1-48$ 之间, MCs 与 TN/TP的相关性不显著. 全年监测的相关性分析结果与不同月份的基本一致.

除蓝藻暴发期, 各个月份的相关性分析表明,水中溶解性 MCs 与 $\mathrm{SS} 、 \mathrm{UV}_{254} 、 \mathrm{TOC} 、$ 蓝藻密度、水温均无显 著相关性 (表 2). 但是全年的相关性结果显示 TOC、蓝藻密度、水温与 MC-LR 具有统计学意义上的负相关 性,与 MC-RR 无显著相关性 (表 1). 因为水中溶解性 MC-LR 浓度表现为在夏秋两季浓度较低,春冬两季浓 度较高, MC-RR 无此规律; 蓝藻密度、水温正好相反; 同时由于温度较高、光照强烈、生物活动剧烈导致 TOC 含量升高 ${ }^{[29]}$, 所以 TOC 含量与温度及蓝藻密度有很好的一致性, 即均与 MC-LR 浓度表现出季节波动的负 相关性. 蓝藻暴发期的相关性分析结果则表明 $\mathrm{UV}_{254}$ 、TOC、SS、蓝藻数量与 MC-LR 呈正相关, 与 MC-RR 无显 著相关性 (表 3). MCs 主要由蓝藻细胞死亡破裂后释放到水体中的 ${ }^{[17]}$, 因此与蓝藻死亡后水体中的 TOC 含 量及 $\mathrm{UV}_{254}$ 值呈正相关, 可能澡细胞中 MCs 种类以 MC-LR 为主 ${ }^{[21]}$, 所以表现出与 MC-LR 极显著相关, 与 MC-RR不显著相关. 可能由于当前死亡蓝藻数量与蓝藻数量相当, 并且以生产 MC-LR 为主 ${ }^{[21]}$, 所以 MC-LR 与 蓝藻数量呈极显著正相关. 同时, 由于处于蓝藻暴发期, SS 的主要组成部分为蓝藻细胞, 所以 MC-LR 也与 SS 呈极显著正相关.

表 1 全年 MCs 与各理化因子相关性分析

Tab. 1 Correlation analysis between microcystin and physicochemical factors of the whole year

\begin{tabular}{|c|c|c|c|c|c|c|}
\hline \multirow{2}{*}{ 理化因子 } & \multicolumn{3}{|c|}{ MC-LR } & \multicolumn{3}{|c|}{ MC-RR } \\
\hline & $r$ & $P$ & $n$ & $r$ & $P$ & $n$ \\
\hline $\mathrm{TP}$ & $0.576^{* *}$ & $<0.01$ & 68 & 0.146 & 0.224 & 71 \\
\hline TSP & 0.236 & 0.051 & 69 & 0.158 & 0.182 & 73 \\
\hline SRP & 0.176 & 0.148 & 69 & 0.205 & 0.081 & 73 \\
\hline $\mathrm{TN}$ & $0.758 * *$ & $<0.01$ & 69 & 0.108 & 0.362 & 73 \\
\hline $\mathrm{NO}_{3}-\mathrm{N}$ & $0.273^{*}$ & 0.023 & 69 & -0.050 & 0.676 & 73 \\
\hline $\mathrm{NO}_{2}-\mathrm{N}$ & $0.772 * *$ & $<0.01$ & 67 & 0.074 & 0.539 & 71 \\
\hline $\mathrm{NH}_{3}-\mathrm{N}$ & $0.469^{* *}$ & $<0.01$ & 66 & 0.047 & 0.708 & 67 \\
\hline $\mathrm{TN} / \mathrm{TP}$ & -0.109 & 0.374 & 68 & -0.085 & 0.483 & 71 \\
\hline $\mathrm{UV}_{254}$ & $0.730^{* *}$ & $<0.01$ & 69 & -0.044 & 0.709 & 73 \\
\hline TOC & $0.289^{* *}$ & 0.016 & 69 & -0.067 & 0.574 & 73 \\
\hline SS & $0.611^{* *}$ & $<0.01$ & 69 & 0.020 & 0.867 & 73 \\
\hline 蓝藻密度 & $0.694^{* *}$ & $<0.01$ & 69 & 0.055 & 0.644 & 73 \\
\hline
\end{tabular}

* 显著相关 $(P<0.05) ; * *$ 极显著相关 $(P<0.01)$ 
表 2 不同时期 MCs 与 TN 的相关性分析

Tab. 2 Correlation analysis between MCs and TN at different times

\begin{tabular}{ccccccccc}
\hline & \multicolumn{7}{c}{ 时间 (年-月-日) } \\
\cline { 2 - 8 } & & $2009-4-25$ & $2009-5-24$ & $2009-7-10$ & $2009-9-1$ & $2009-9-25$ & $2009-11-25$ & $2010-1-16$ \\
\hline \multirow{2}{*}{ MC-LR } & $r$ & $0.691^{*}$ & $0.709^{*}$ & $0.758^{* *}$ & -0.173 & $0.635^{*}$ & 0.273 & $0.645^{*}$ \\
& $P$ & 0.019 & 0.022 & $<0.01$ & 0.611 & 0.036 & 0.416 & 0.032 \\
& $n$ & 11 & 10 & 69 & 11 & 11 & 11 & 11 \\
MC-RR & $r$ & $0.716^{*}$ & $0.758^{*}$ & 0.108 & -0.018 & 0.533 & $0.645^{*}$ & $0.656^{*}$ \\
& $P$ & 0.013 & 0.011 & 0.362 & 0.958 & 0.091 & 0.032 & 0.028 \\
& $n$ & 11 & 10 & 73 & 11 & 11 & 11 & 11 \\
\hline
\end{tabular}

*显著相关 $(P<0.05)$; **极显著相关 $(P<0.01)$

表 3 蓝藻暴发期 MCs 与各理化因子相关性分析

Tab. 3 Correlation analysis between microcystin and physicochemical factors during cyanobacteria blooms

\begin{tabular}{|c|c|c|c|c|c|c|}
\hline \multirow{2}{*}{ 理化因子 } & \multicolumn{3}{|c|}{ MC-LR } & \multicolumn{3}{|c|}{ MC-RR } \\
\hline & $r$ & $P$ & $n$ & $r$ & $P$ & $n$ \\
\hline $\mathrm{TP}$ & $0.576^{* *}$ & $<0.01$ & 68 & 0.146 & 0.224 & 71 \\
\hline TSP & 0.236 & 0.051 & 69 & 0.158 & 0.182 & 73 \\
\hline SRP & 0.176 & 0.148 & 69 & 0.205 & 0.081 & 73 \\
\hline $\mathrm{TN}$ & $0.758^{* *}$ & $<0.01$ & 69 & 0.108 & 0.362 & 73 \\
\hline $\mathrm{NO}_{3}-\mathrm{N}$ & $0.273^{*}$ & 0.023 & 69 & -0.050 & 0.676 & 73 \\
\hline $\mathrm{NO}_{2}-\mathrm{N}$ & $0.772^{* *}$ & $<0.01$ & 67 & 0.074 & 0.539 & 71 \\
\hline $\mathrm{NH}_{3}-\mathrm{N}$ & $0.469^{* *}$ & $<0.01$ & 66 & 0.047 & 0.708 & 67 \\
\hline $\mathrm{TN} / \mathrm{TP}$ & -0.109 & 0.374 & 68 & -0.085 & 0.483 & 71 \\
\hline $\mathrm{UV}_{254}$ & $0.730^{* *}$ & $<0.01$ & 69 & -0.044 & 0.709 & 73 \\
\hline TOC & $0.289^{* *}$ & 0.016 & 69 & -0.067 & 0.574 & 73 \\
\hline SS & $0.611^{* *}$ & $<0.01$ & 69 & 0.020 & 0.867 & 73 \\
\hline 蓝藻密度 & $0.694^{* *}$ & $<0.01$ & 69 & 0.055 & 0.644 & 73 \\
\hline
\end{tabular}

*显著相关 $(P<0.05) ; * *$ 极显著相关 $(P<0.01)$

\section{2 MCs 与环境因子的回归分析}

全年水中溶解性 MCs 逐步回归分析表明, 对于 MC-LR 最好的回归模型只有 TN 和 SRP 进人, 其 $R^{2}$ 为 0.362 , 拟合度较差; 而 MC-RR 只有 $\mathrm{NO}_{2}-\mathrm{N}$ 进人模型, 其 $R^{2}$ 为 0.332 , 拟合度也较差. 以现有的环境因子难以 对水体中溶解性 MCs 的浓度进行预测, 有研究表明水体中溶解态 MCs 的时空分布受风向、风速的影响较 $大^{[30]}$, 增加风向、风速及水量等物理因子可能能够预测全年水体中溶解性 MCs 的浓度.

\section{3 结论}

对太湖全湖 9 个点位藻毒素为期一年的监测发现表层与底层藻毒素含量几乎没有差别, MC-LR 与 MC$\mathrm{RR}$ 的浓度大小则表现为除 $8 、 9$ 月份以外 MC-LR 均大于 MC-RR, 藻毒素在各点分布不均, 在时间上表现为 冬季浓度较高, 在夏季, 一天之内藻毒素浓度没有明显的变化规律. 北部 N1 和 N5 点浓度在一年内变化较大, 在蓝藻暴发后藻毒素浓度明显增加, 而其他点均未发现有此规律. 通过对藻毒素与一些环境因子的相关性分 析发现, 这些环境因子共同作用影响蓝藻产毒量和藻毒素在环境中的归趋从而影响藻毒素的含量, 是一个非 常复杂的过程. 其中风向、风速以及水量等物理因素可能对藻毒素的浓度及其时空分布起着非常重要的作用. 致谢: 感谢南京大学水处理与水环境修复教育部工程研究中心在水样采集和样品分析过程中提供的大力 协助.

\section{4 参考文献}

[ 1 ] Falconer IR. Tumor promotion and liver injury caused by oral consumption of cyanobacteria. Environ Toxic Water, 1991, 6(2) : 177-184.

[2 ] Dowson RD. The toxicity of microcystins. Toxicon, 1998, 36 (7) : 953-962. 
[ 3 ] Baldia SF, Conaco MCG, Nishijima T et al. Microcystin production during algal bloom occurrence in Laguna de Bay, the Philippines. Fisheries Sci, 2003, 69: 110-116.

[ 4 ] Watanabe MF. Toxic Microcystis. Boca Raton: CRC Press, 1996: 35-36.

[ 5 ] Figueiredo DR, Azeiteiro UM, Esteves SM et al. Microcystin-producing bloom-a serious global public health issue. Ecotox Environ Safe, 2004, 59(2) : 151-163.

[ 6 ] Vasconcelos VM, Carmichael WW, Croll B et al. Hepatotoxic microcystin diversity in cyanobacterial blooms collected in portuguese freshwaters. Water Res, 1996, 30(10) : 2377-2389.

[ 7 ] 国家环境保护总局主编. 水和废水监测分析方法 (第四版). 北京: 中国环境科学出版社, 2002:247-246,254-279, 106-108,70-671.

[8] 刘约权主编. 现代仪器分析. 北京: 高等教育出版社,2001:122-159.

[9] 全国渔业自然资源调查和淡水专业组. 内陆水域渔业自然资源调查试行规范. 全国渔业自然资源调查和淡水专业 组, $1980: 12-15$.

[10］毛新伟,徐 枫,徐 涁等. 水质及富营养化变化趋势分析. 水资源保护,2009,25(1):48-51.

[11] 宋立荣,陈 伟. 水华蓝藻产毒的生物学机制及毒素的环境归趋研究进展. 湖泊科学,2009,21(6):749-757.

[12] Chen W, Song LR, Peng L et al. Reduction in microcystin concentrations in large and shallow lakes: water andsedimentinterface contributions. Water Res, 2008, 42 : 763-773.

[13] Briand E, Yéprémian C, Humbert JF et al. Competition between microcystin-and non-microcystin-producing Planktothrix agardhii (cyanobacteria) strains under different environmental conditions. Environ Microbiol, 2008, 10(12) : 3337-3348.

[14] Ye WJ, Liu XL, Tan J et al. Diversity and dynamics of microcystin-Producing cyanobacteria in China's third largest lake, Lake Taihu. Harmful Algae, 2009, 8: 637-644.

[15] Kim SG, Joung SH, Ahn CY et al. Annual variation of Microcystis genotypes and their potential toxicity in water and sediment from a eutrophic reservoir. FEMS Microbiol Ecol, 2010, 74: 93-102.

[16] Shi L, Carmichael WW, Miller I. Immuno-gold localization of hepatotoxins in cyanobacterial cells. Arch Microbiol, 1995, $163: 7-15$

[17] Caemichael WW. The toxicity of cyanobacteria. Sci Am, 1994, 270(1): 64-70.

[18 ] Young FM, Thomson C, Metcalf JS et al. Immunogold localisation of microcystins in cryosectioned cells of Microcystis. J Struct Biol, 2005 , 151 : 208-214.

[19] Sivonen K. Effects of light, temperature, nitrate, orthophosphate, and bacteria on growth of and hepatotoxin production by Oscillatoria agardhii strains. Appl Environ Microbiol, 1990, 56: 2658-2666.

[20] Negri AP, Jones GJ, Blackburn SI et al. Effect of culture and bloom development and of sample storage on paralytic shellfish poisons in the cyanobacterium Anabaena circinalis. J Phycol, 1997, 33 : 26-35.

[21] Kardinaal WEA, Janse I, Kamst-van Agterveld M et al. Microcystis genotype succession in relation to microcystin concentrations in freshwater lakes. Aquat Microb Ecol, 2007, 48: 1-12.

[22] Botes DP, Wessels PL, Kruger H et al. Structural studies on cyanoginosins-LR, -YR, -YA and -YM, peptide toxins from Microcystis aeruginosa. J Chem Soc Perk T 1, 1985, 1: 2747-2748.

[23] 代瑞华,刘会娟,曲久辉等. 氮磷限制对铜绿微囊藻生长和产毒的影响. 环境科学学报,2008,28(9):1739-1744.

[24] 张杭君,张建英,陈英旭等. 微囊藻毒素含量与自然水体环境影响因子的相关性. 环境科学, 2006, 27 (10): 1969-1973.

[25] 连 民,陈传玮,俞顺章等. 淀山湖夏季微囊藻毒素分布状况及其影响因素. 中国环境科学, 2000,20(4):323-327.

[26] Utkilen H, Gjolme N. Iron-stimulated toxin production in microcystis aeruginosa. Appl Environ Microbiol, 1995, 61(2) : 797-800.

[27 ] Oh HM, Lee SJ, Jang MH et al. Microcystin production of microcystis aeruginosa in a Phosphorus-limited chemostat. Appl Environ Microbiol, 2000, 66(1): 176-179.

[28] 张 玮, 林一群, 郭定芳等. 不同氮、磷浓度对铜绿微囊藻生产、光合及产毒的影响. 水生生物学报, 2006, 30(3): 318-322.

２9］江志坚,黄小平,张景平. 大亚湾海水中总有机碳的时空分布及其影响因素. 海洋学报,2009,31 (1):91-98.

[30 ] 许秋瑾,高 光,陈伟民. 太湖微囊藻毒素与湖泊物理因素之间的关系. 中国环境监测,2004,20(1):12-15. 